F. Reprod. Fert. (1970) 21, 29 35

\title{
IMMUNOLOGICAL ASPERMATOGENESIS IN MAN
}

\section{RESPONSE TO CORTICOSTEROIDS IN CASES OF NON- OBSTRUCTIVE AZOOSPERMIA WITH A POSITIVE BLASTOID TRANSFORMATION TEST}

\author{
F. BASSILI* AND O. S. EL-ALFI $\dagger$ \\ *Family Planning Centre, Ministry of Public Health, Kuwait, and \\ $\dagger$ Unit of Medical Genetics, Sabah Hospital, Kuwait
}

(Received 11th November 1968)

\begin{abstract}
Summary. Corticosteroids were used in the treatment of a group of eighteen non-obstructive azoospermic or severely oligospermic patients showing a positive lymphocyte blastoid transformation test in response to a seminal antigen. A control group of eleven similarly affected patients with negative blastoid transformation tests was given the same treatment. Response was noticed in nine of the positive test group $(50 \%)$, and one patient's wife conceived. None of the control group showed any response.

The value of this trial therapy is discussed.
\end{abstract}

\section{INTRODUCTION}

An immunological reaction was demonstrated in a group of infertile males with non-obstructive oligospermia or azoospermia (El-Alfi \& Bassili, 1970). The present paper reports the findings in the same group of patients when subjected to corticosteroid therapy.

\section{MATERIAL}

Eighteen of the twenty-one patients who showed a positive lymphocyte blastoid transformation test were given corticosteroids orally. The remaining three patients were not available at the time of the trial. The ages of the men receiving corticosteroids ranged from 25 to 50 years, with an average of 38.5 years, and the number of years during which they had been known to be infertile ranged from 1 to 29 with an average of 8.7 years. Thirteen patients were completely azoospermic during the period before treatment, while the remaining five gave reports varying from severe oligospermia to azoospermia before the trial therapy.

Six patients had a history of previous testicular injury in the form of trauma, biopsy or orchitis. Twelve had bilateral small, soft testes while the remaining six had testes of normal size and consistency (Table 1). 
As controls, eleven patients were chosen at random from the group of patients with non-obstructive azoospermia who had a negative blastoid transformation test. Their ages ranged from 20 to 55 years with an average of

TABLE 1

HISTORY OF TESTIGULAR INJURY AND SIZE AND CONSISTENCY OF TESTES IN EIGHTEEN INFERTILE MEN WITH POSITIVE LYMPHOCYTE BLASTOID TRANSFORMATION TESTS TREATED WITH CORTICOSTEROID

\begin{tabular}{c|ccc|cc}
\hline & \multicolumn{4}{|c|}{} & \multicolumn{2}{c}{ History of testicular injury } & \multicolumn{2}{c}{$\begin{array}{c}\text { Size and consistency of } \\
\text { testes }\end{array}$} \\
Case No. & $\begin{array}{c}\text { Infection } \\
\text { Trauma }\end{array}$ & Biopsy & Small and soft & Normal \\
\hline 8 & - & - & + & - & + \\
21 & - & - & + & + & - \\
25 & - & - & - & + & - \\
27 & - & - & - & + & - \\
28 & - & - & - & + & - \\
48 & - & - & - & + & - \\
50 & - & + & - & + & - \\
51 & - & - & - & + & - \\
54 & - & - & - & + & - \\
85 & - & - & - & - & + \\
113 & + & - & - & + & - \\
120 & + & - & - & + & - \\
121 & - & - & - & - & + \\
123 & - & - & - & - & + \\
131 & + & - & - & - & + \\
134 & - & - & - & + & + \\
145 & - & - & - & - & + \\
160 & - & - & - & + & - \\
\hline
\end{tabular}

TABLE 2

HISTORY OF TESTIGULAR INJURY AND SIZE AND GONSISTENCY OF TESTES IN ELEVEN GONTROL PATIENTS

\begin{tabular}{|c|c|c|c|c|c|}
\hline \multirow[b]{2}{*}{ Case No. } & \multicolumn{3}{|c|}{ History of testicular injury } & \multicolumn{2}{|c|}{$\begin{array}{c}\text { Size and consistency of } \\
\text { testes }\end{array}$} \\
\hline & Infection & Trauma & Biopsy & Small and soft & Normal \\
\hline 42 & - & - & - & + & - \\
\hline 62 & - & - & - & + & - \\
\hline 72 & + & - & - & + & - \\
\hline 74 & - & - & - & - & + \\
\hline 82 & + & - & - & + & - \\
\hline 86 & + & - & - & + & - \\
\hline 96 & - & - & - & - & + \\
\hline 107 & - & - & - & + & - \\
\hline 114 & - & - & - & - & + \\
\hline 116 & - & - & - & - & + \\
\hline 126 & - & - & - & + & - \\
\hline
\end{tabular}

34.2 years and they had been known to be infertile for 1 to 25 years (average 9.5 years). Six patients gave a history of complete azoospermia, and the remaining five a history of alternating oligospermia and azoospermia, before the start of the trial. A history of previous testicular injury was recorded for three of 
Immunological aspermatogenesis in man. II

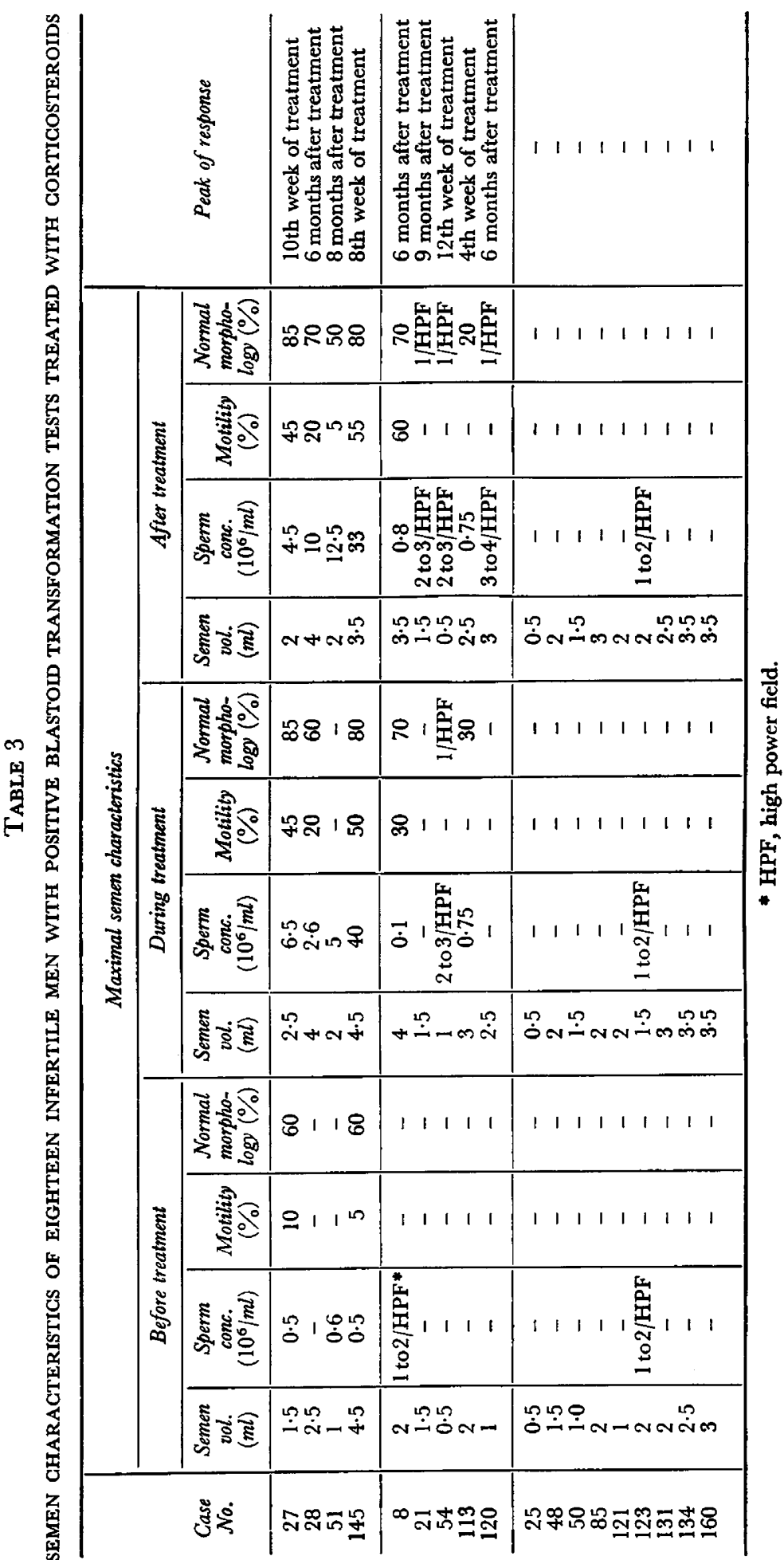


them. Seven patients had bilateral small, soft testes and in the remaining four, the testes were normal (Table 2).

\section{Treatment}

The corticosteroid dose (prednisone, 5-mg tablets) was standardized for all patients by giving $40 \mathrm{mg}$ /day for 2 weeks, followed by a gradual reduction over a 9-week period. The prednisone used was a synthetic product, and contained no traces of testosterone.

The patients were checked carefully for hypertension, peptic ulcer, chest affections, diabetes, Cushingoid manifestations and any mental troubles before giving prednisone.

All the men were treated as out-patients. They had to report every week for clinical checking, and for semen examination following a 5-day period of continence.

\section{RESULTS}

Of the eighteen patients treated, four showed a good response both during and after treatment, five showed a poor response and nine showed no response.

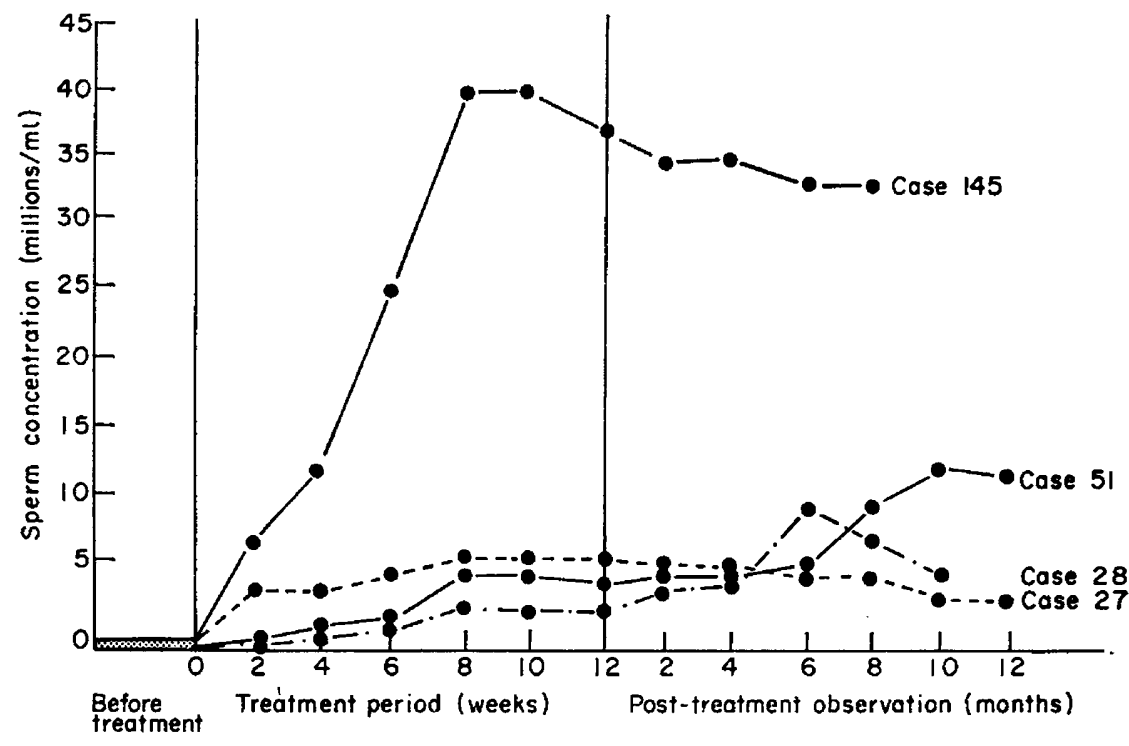

TEXT-FIG. 1. Sperm concentration in four infertile men showing good response to corticosteroid therapy.

Of the four patients showing a good response (Table 3), three continued to maintain good sperm counts 8 to 12 months after the end of treatment. The sperm counts of the fourth patient (Case No. 28), started to decline 10 months after the end of treatment, and he is now azoospermic again (Text-fig. 1).

The motility of the spermatozoa was good ( 40 to $50 \%$ active) in two of these patients, and poor in the remaining two (Table 3). Three patients had alternating azoospermia and severe oligospermia, while the fourth was completely 


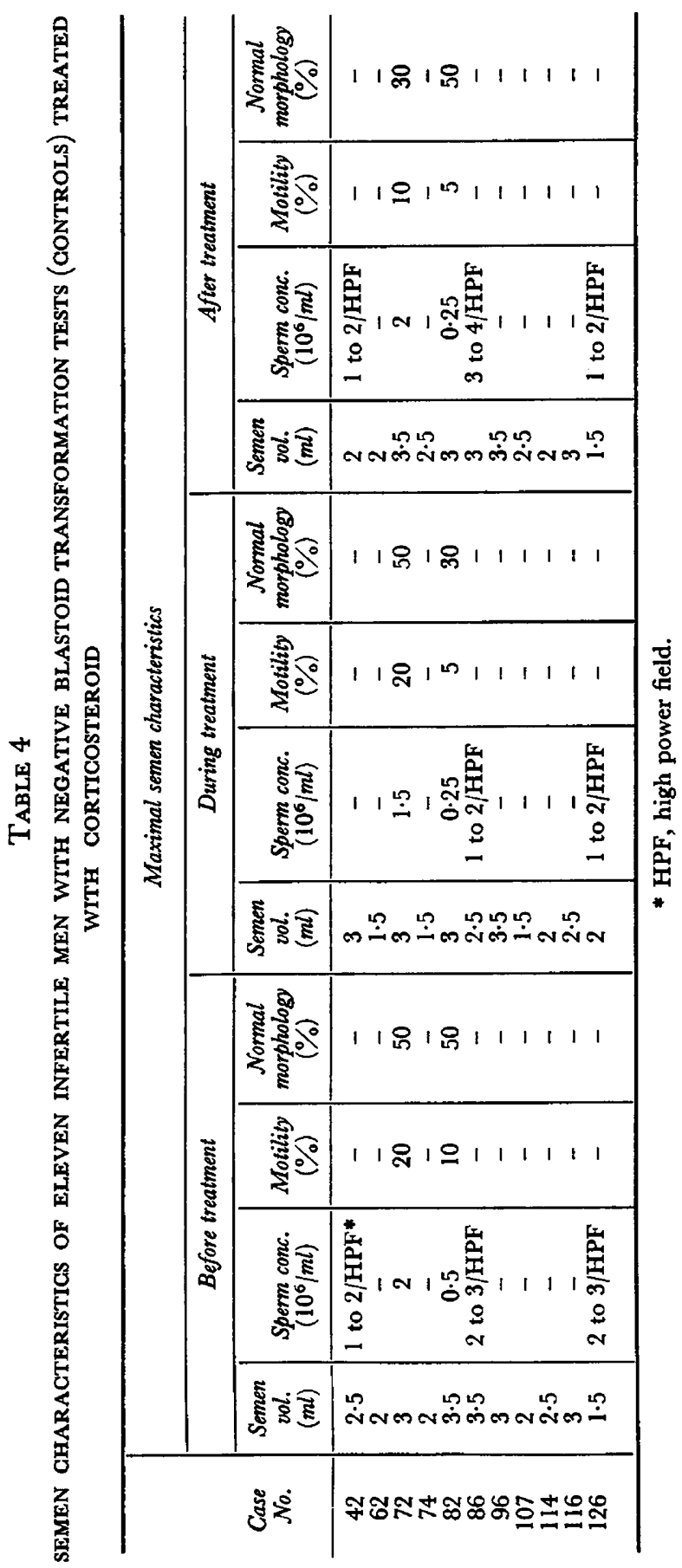


azoospermic before treatment. There was no history of testicular injury in any of these patients but three had bilateral small, soft testes. In the fourth (Case No. 145), the size and consistency were normal. This patient showed the best results. His total sperrn count rose from 2.25 million before, to 180 million during treatment and his wife became pregnant. Her first missed period occurred 3 months after the onset of treatment.

The five patients who responded poorly to treatment (Table 3) did show a slight increase in their sperm counts, but their spermatozoa were mostly sluggish or immotile. This small improvement appeared during treatment in two patients, and after treatment in three patients (Table 3). Four had azoospermia, and one severe oligospermia before the start of treatment. Four gave a history of testicular injury (Table 1), and the testes were soft and small in four and of normal size and consistency in one.

Of the remaining nine patients with a positive blastoid transformation test who showed no response to corticosteroid therapy, eight had azoospermia and one oligospermia before the start of therapy. Two had a history of testicular injury. Bilateral small, soft testes were found in five and testes of normal size and consistency in four (Table 1).

None of the control patients showed any improvement in their pre-treatment counts (Table 4).

There was no significant change in the accessory organs of any of the treated patients.

\section{DISCUSSION}

Treatment of infertility resulting from an auto-immune disorder in the male has not yet been greatly successful. Wilson (1956) tried donor insemination which resulted in a successful pregnancy. Rozin (1960). suggested the in vitro substitution of normal centrifuged acellular seminal plasma from donors for the agglutinin-rich seminal plasma of infertile husbands who still have good sperm counts, but an azoospermic or severely oligospermic husband cannot be helped by such a line of treatment. The present group of eighteen patients all had non-obstructive azoospermia or severe oligospermia, and all gave evidence of an immunological disorder manifested by a positive lymphocyte blastoid transformation reaction to a seminal antigen. The increase in sperm count recorded here for nine $(50 \%)$ patients in response to corticosteroid therapy gave some encouragement to a number of infertile males for whom, as yet, there had been no successful treatment. The good response of some patients to the corticosteroid therapy supports the hypothesis of auto-immune aspermatogenesis as a cause of infertility in man and the absence of any response in the controls strongly supports the reliability of the blastoid transformation test in the detection of such cases (El-Alfi \& Bassili, 1970).

It is believed that in the case of the three patients who showed a good rise in their sperm counts (Case Nos. 27, 28 and 51) but whose wives did not become pregnant, and probably also in the case of some of the patients who showed a poor response to corticosteroids but some increase in sperm count, artificial insemination of the husband's semen (AIH) is worth a trial, particularly since the rise in the sperm counts might not be long lasting. The sperm 
count of one patient (Case No. 28) showed a gradual rise to 10.4 million during treatment, and 40 million 6 months afterwards, but the counts gradually declined to zero level 12 months after the termination of treatment. Another point in favour of AIH is that the rise in the total sperm count may not reach a level that would allow normal conception.

It is difficult, in a group of patients like the present one, to give an individual prognosis. Changes in the degree of blastoid transformation could not be correlated with rise in sperm count. Corticosteroid therapy suppressed the blastoid transformation reactions whether or not the testicular tubules responded to suppression of the auto-immune process. Similarly, a changing titre of circulating antibodies could not be taken as a criterion for prognosis. It appears from Table 3 that individuals who are severely oligospermic before therapy probably have a better chance of responding to corticosteroids than those with a history of prolonged azoospermia. However, some of those with prolonged complete azoospermia did respond, while other similar cases failed to respond. It is possible that in the latter group, the testicular tubules were so damaged that even after the suppression of the auto-immune process, spermatogenesis could not be resumed.

\section{REFERENCES}

EL-ALFI, O. S. \& BASSILI, F. (1970) Immunological aspermatogenesis in man. I. Blastoid transformation of lymphocytes in response to seminal antigen in cases of non-obstructive azoospermia. $\mathcal{J}$. Reprod. Fert. 21, 23.

Rozin, S. (1960) Studies on seminal plasma. I. The role of seminal plasma in motility of spermatozoa. Fert. Steril. 11, 278.

Wilson, L. (1956) Sperm agglutination due to auto-antibodies. A new cause of sterility. Fert. Steril. 7, 262. 